superoxide release in atrial samples of patients with post-operative AF but had no effect in patients with permanent AF. Similarly, atorvastatin did not induce a mevalonate-reversible changes in the atrial BH4 concentration and NOS uncoupling in neither group.

Conclusions Together, these findings indicate that upregulation of NOX2-NADPH oxidases is an early but transient event in the natural history of AF, as mitochondrial oxidases and uncoupled NOS account for the statin-resistant increase in atrial superoxide production in permanent AF. Variation in atrial sources of reactive oxygen species with the duration and substrate of AF may explain the reported variability in the effectiveness of statins in the prevention and management of AF.

\section{TISSUE FACTOR PATHWAY INHIBITOR REGULATES ANGIOGENESIS INDEPENDENTLY OF TISSUE FACTOR VIA INHIBITION OF VASCULAR ENDOTHELIAL GROWTH FACTOR SIGNALLING}

doi:10.1136/heartjnl-2011-300198.143

${ }^{1} \mathrm{E}$ W Holroyd, ${ }^{2} \mathrm{~K}$ Larsen, ${ }^{2} \mathrm{R}$ G Vile, ${ }^{2} \mathrm{D}$ Mukhopadhyay, ${ }^{2} \mathrm{R} \mathrm{D}$ Simari. ${ }^{1}$ Queen Elizabeth Hospital, Birmingham, UK; ${ }^{2}$ Mayo Clinic, Rochester, Minnesota, USA

Introduction The biological systems of coagulation and angiogenesis show considerable interdependence. Proteases and inhibitors within the tissue factor (TF) pathway of coagulation have emerged as potential regulators of angiogenesis. Tissue factor pathway inhibitor (TFPI), as the primary physiological inhibitor of tissue factor (TF)mediated coagulation, is ideally situated to modulate the proangiogenic effects of TF. However, TFPI may also have effects on angiogenesis independent of its anti-TF ability.

Methods We determined the effects of altered TFPI expression on the regulation of angiogenesis in vivo using genetically-modified murine models of vascular overexpression (SM22áTFPI strain) and endothelial-specific deletion of the TF-binding domain of TPFI (Tie2TFPI). We then defined the mechanism of these effects in vitro using Human Umbilical Vein Endothelial Cells (HUVECs) overexpressing TFPI or via exogenous addition of TFPI-derived peptides in assays of angiogenesis.

Results Vascular-directed over-expression of TFPI (SM22áTFPI strain) inhibited angiogenesis in vivo (Abstract 143 figure 1). SM22áTFPI showed significantly impaired recovery from ischaemia in the hindlimb ischaemia model after 3 days $(\mathrm{p}<0.05, \mathrm{n}=5$ per group), which persisted throughout the experiment. Survival (until 1-cm tumour dimension) of SM22áTFPI mice vs wild-type control (median survival $14 \mathrm{cf}$. 10 days) following s.c. B16 melanoma injection $\left(n=7\right.$ per group, $\left.\chi^{2}=4.325,{ }^{*} p<0.05\right)$. Endothelial-specific deletion of the TF-binding domain of TFPI failed to reveal a proangiogenic phenotype. This led us to suspect that the anti-angiogenic action of TFPI may be independent of TF. Systemic delivery of the murine TFPI carboxyl-terminus (mTFPIct) replicated the effects of endogenous overexpression. In vitro, overexpression of TFPI inhibited endothelial cell tube formation on Matrigel and migration using an injury migration model. Human TFPIct (hTFPIct) inhibited tube formation and migration through inhibition of Vascular Endothelial Growth Factor Receptor-2 (VEGFR2) tyrosine-951 phosphorylation, a key event in migration. hTFPIct did not inhibit VEGF121-induced migration, which lacks the heparin-binding domain of VEGF165. Utilising the chimeric receptor, EGDR, which contains the extracellular domain of epidermal growth factor (EGF) and the intracellular domain of VEGFR2/KDR, a direct effect of TFPIct on the intracellular domain of VEGFR2 was excluded (Abstract 143 figure 2) TFPIct did not block phosphorylation of EGDR when stimulated with EGF.
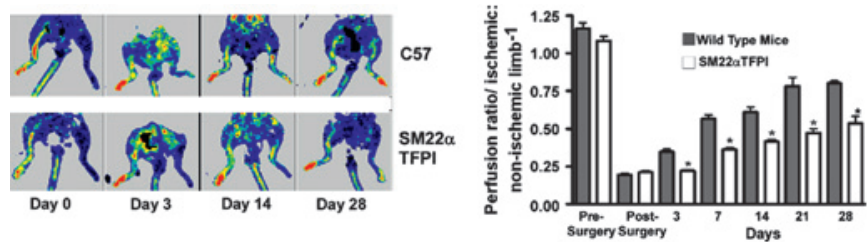

Abstract 143 Figure 1
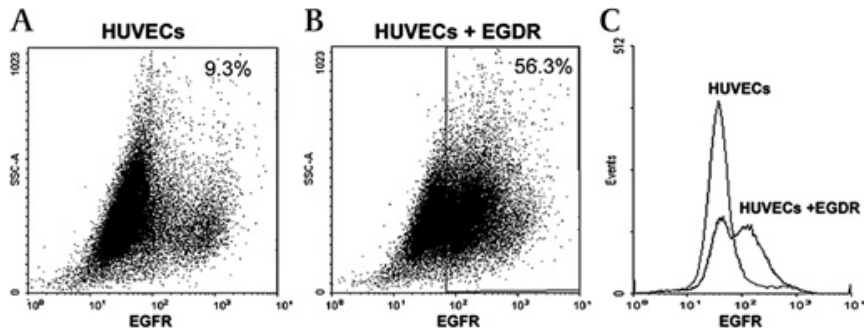

D

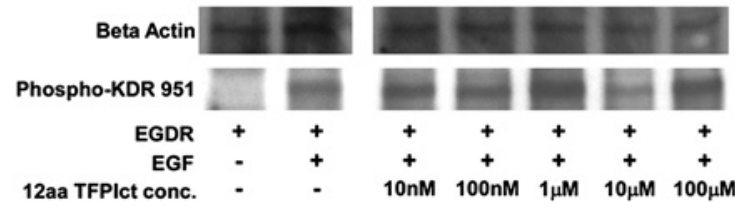

Abstract 143 Figure 2

Conclusion Angiogeneis is a key biological system in health and disease; enabling cells in a hypoxic environment to stimulate new blood vessel growth. These data demonstrate, both in vivo and in vitro, an inhibitory role for TFPI in angiogenesis that is TF-independent. In addition to it classical role as a TF-antagonist, TFPI, via TFPIct, interferes with the interaction of VEGF165 with the extracellular domain of VEGFR2, thereby limiting angiogenesis.

\section{4 A DRUGGABLE INHIBITOR OF CARDIAC HYPERTROPHY
IDENTIFIED THROUGH AN INNOVATIVE CHEMICAL LIBRARY
SCREEN}

doi:10.1136/heartjnl-2011-300198.144

R Abou Leisa, T M A Mohamed, D Oceandy, S Prehar, M Zi, F Baudoin, L Neyses, E J Cartwright. Manchester University, Manchester, UK

Cardiac hypertrophy is a prerequisite for the development of heart failure. It currently affects almost one million people in the UK. Few effective anti-hypertrophic agents with druggable properties have been identified. Recently, our group showed that plasma membrane calcium ATPase isoform 4 (PMCA4) knockout mice showed a reduced response to hypertrophic stress prompting us to hypothesise that a novel PMCA4 specific inhibitor would modify the development of cardiac hypertrophy. A library of 1280 medically optimised compounds was screened using a novel in vitro assay which measures the Ca2 + dependent ATPase activity of PMCA4. The compound AP2 was identified, which inhibited PMCA4 activity with high affinity $\left(\mathrm{IC}_{50}=300 \mathrm{nM}\right.$ ) but not other PMCAs (PMCA1, PMCA2 and PMCA3) or related ATPases which are expressed in the heart including the sarcoplasmic reticulum calcium ATPase and $\mathrm{Na} / \mathrm{K}$ ATPase. In isolated neonatal rat cardiomyocytes (NRCM), AP2 showed dose dependent inhibition of phenylephrine-induced hypertrophy, indicated by an $85 \%$ reduction in cell surface area as well as in BNP activity. In vivo studies showed that AP2 (5 mg/kg body weight/day IP) significantly reduced pressure-overload induced hypertrophy following 2 weeks transverse aortic constriction (TAC) (heart weight/tibia length (mg/ $\mathrm{mm}$ ): sham, $5.5 \pm 0.3$, vehicle treated TAC mice, $8.7 \pm 0.2$, AP2 treated TAC mice, $7.0 \pm 0.5, \mathrm{n}=10$ in each group, $\mathrm{p}<0.01)$. AP2 treated TAC 
mice showed a significant reduction in the cardiomyocyte cross sectional area (sham, $267 \pm 3.4 \mu \mathrm{m}^{2}$, vehicle treated TAC mice, $480 \pm 5.8 \mu \mathrm{m}^{2}$, AP2 treated TAC mice, $\left.319 \pm 3.9 \mu \mathrm{m}^{2}\right)$. A significant reduction in the expression of the hypertrophic marker ANP and BNP and in the percentage of fibrosis was also observed in these mice compared with vehicle treated TAC mice. AP2 treatment led to a significant reduction in the expression of the bona fide calcineurin target RCAN1.4 and a reduction in the NFAT phosphorylation level in vivo and the NFAT transcriptional activity in vitro. In conclusion, we have identified AP2 as a novel PMCA4 specific inhibitor and shown its potential to modify the development of cardiac hypertrophy likely through inhibition of calcineurin/NFAT signalling. This compound has drug-like properties and thus lays the basis for a novel approach for treating cardiac hypertrophy and failure through PMCA4 inhibition.

\section{CHARACTERISATION OF FRACTIONATED ATRIAL ELECTROGRAMS CRITICAL FOR MAINTENANCE OF AF: A RANDOMISED CONTROLLED TRIAL OF ABLATION STRATEGIES (THE CFAE AF TRIAL)}

doi:10.1136/heartjnl-2011-300198.145

R J Hunter, I Diab, M Tayebjee, L Richmond, S Sporton, M J Earley, R J Schilling. Barts and The London NHS Trust, London

Introduction Targeting complex fractionated atrial electrograms (CFAE) in the ablation of atrial fibrillation (AF) may improve outcomes, although whether this is by eliminating focal drivers or simply de-bulking atrial tissue is unclear. It is also uncertain what electrogram morphology should be ablated. This randomised study aimed to determine the impact of ablating different CFAE morphologies compared to normal electrograms (ie, de-bulking normal tissue) on the cycle length of persistent AF (AFCL).

Methods After pulmonary vein isolation CFAE were targeted systematically throughout the left then right atrium, until termination of AF or abolition of CFAE prior to DC cardioversion. $10 \mathrm{~s}$ electrograms were classified by visual inspection according to a validated scale, with Grade 1 being most fractionated and grade 5 normal. Patients were randomised to have CFAE grades eliminated sequentially, from grade 1 to 5 (group 1) or grade 5 to 1 (group 2). Because grade 5 electrograms were considered normal, only 5 were ablated. Mean AFCL was determined manually over 30 cycles from bipolar electrograms recorded at the left and right atrial appendages before and after each CFAE was targeted. Lesions were regarded as individual observations, and a resultant increase in mean AFCL $\geq 5 \mathrm{~ms}$ was regarded as significant. The randomised strategy first controlled for any cumulative effect of ablation on AFCL, and second allowed assessment of the order of ablation on the number of CFAE lesions required.

Results 20 patients were randomised. The CFAE grade determined by rapid visual inspection for the 968 electrograms targeted agreed with that at off-line manual measurement in $92.7 \%(\mathrm{~K}=0.91)$. AFCL increased after targeting $49.5 \%$ of grade 1 CFAE, $33.6 \%$ of grade 2 , $12.8 \%$ of grade $3,33.0 \%$ of grade 4 , and $8.2 \%$ of grade 5 CFAE ( $p<0.0001$ for grades 1,2 , and 4 vs 5,3 vs 5 not significant). Binary logistic regression confirmed the effect of CFAE grade, but showed no effect of electrogram amplitude, location in the left or right atrium, or the order in which CFAE were targeted. There was no difference between groups in the number of grade 1 or 2 CFAE encountered, but there were fewer grade 3 and 4 CFAE in group 2 than group 1 (both $\mathrm{p}<0.01)$, translating to fewer CFAE targeted per patient in group 1 compared to group 2 ( $37 \pm 14$ and $58 \pm 18$ respectively; $\mathrm{p}=0.015)$.

Conclusion Targeting CFAE is not simply atrial de-bulking. Ablating certain grades of CFAE caused AFCL prolongation, suggesting they are more important in maintaining AF. Targeting these CFAE may reduce unnecessary left atrial destruction. (ClinicalTrials.gov number, NCT00894400).

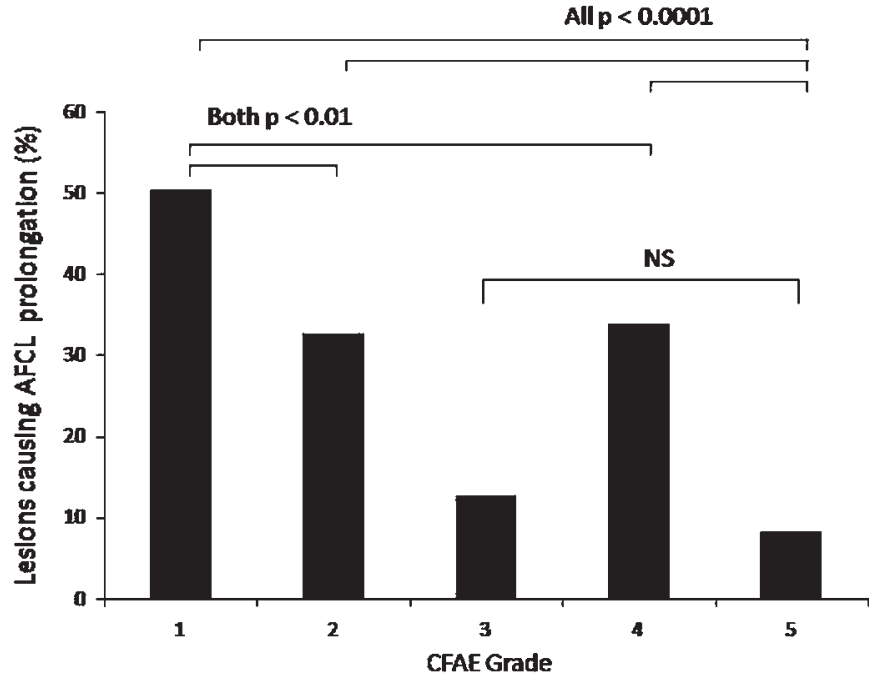

Abstract 145 Figure 1 Impact of CFAE grade on the proportion of lesions causing AF cycle length prolongation.

\section{IS THERE AN ASSOCIATION BETWEEN THROMBOGENESIS MARKERS AND ATRIAL FIBRILLATION BURDEN IN PACEMAKER POPULATION?}

doi:10.1136/heartjnl-2011-300198.146

C W Khoo, S Krishnamoorthy, G Dwivedi, B Balakrishnan, HS Lim, G Y H Lip. University Department of Medicine Centre for Cardiovascular Sciences, City Hospital, Birmingham, UK

Background and Objectives Contemporary pacemaker devices are able to quantify atrial high-rate episodes (AHREs) and atrial fibrillation burden (AFB) accurately. In this study, we aim to assess the relationship of thrombogenesis markers in association with AHREs and AFB.

Methods We studied 87 patients with dual-chamber pacemaker. Patients on warfarin were excluded. AHREs were defined as atrialrate $\geq 220$ beats $/ \mathrm{min}$ and $\geq 5$ minutes. AFB and percentage of cumulative pacing were derived from pacemaker diagnostics. Plasma levels of von Willebrand factor (vWf), tissue factor (TF), soluble P-selectin (P-sel) and D-dimer (DDM) were analysed using ELISA.

Results Baseline characteristics and co-morbidities were comparable between groups (Abstract 146 table 1). Patients with AHREs had significantly higher cumulative percentage ventricular pacing $(p=0.012)$. There were no significant differences in levels of vWf, TF, P-sel and DDM between patients with and without AHREs. The AFB ranged from 0 to $99 \%$ in AHRE group. TF ( $r=0.516, p=0.086)$, P-sel $(r=0.795, p<0.001)$ and DDM $(r=0.643, p=0.045)$ correlated with AFB. On linear regression analysis, both P-sel and DDM were independently associated with AFB $(p<0.05)$.

\section{Abstract 146 Table 1}

\begin{tabular}{llll}
\hline & No AHRE $(\mathbf{n}=70)$ & AHRE $(\mathbf{n}=17)$ & p value \\
\hline Age, years & $71.0 \pm 11.6$ & $75.4 \pm 8.8$ & 0.096 \\
Hypertension, (\%) & $38,(54)$ & $12,(71)$ & 0.116 \\
Antiplatelet, (\%) & $53,(76)$ & $14,(82)$ & 0.739 \\
Percentage atrial pacing & $34.6(6.8-81.5)$ & $22.1(6.9-65.0)$ & 0.414 \\
Percentage ventricular pacing & $21.9(1.8-99.0)$ & $98.6(41.0-99.9)$ & 0.012 \\
vWf, IU/dl & $94.2 \pm 16.2$ & $93.9 \pm 33.7$ & 0.977 \\
TF, ng/ml & $0.2(0.1-0.3)$ & $0.1(0.0-0.2)$ & 0.105 \\
P-sel, ng/ml & $47.6 \pm 15.8$ & $63.4 \pm 29.7$ & 0.055 \\
$\mathrm{DDM}, \mathrm{ng} / \mathrm{ml}$ & $180.0(82.0-390.0)$ & $152.5(82.5-307.5)$ & 0.553 \\
\hline
\end{tabular}

